\title{
LES ÉLECTIONS DE MARS 2008 EN ESPAGNE ET EN CATALOGNE
}

\author{
Joan Marcet et al.
}

ARPoS | Pôle Sud

$2008 / 2-n^{\circ} 29$

pages 9 à 24

\section{ISSN 1262-1676}

Article disponible en ligne à l'adresse:

http://www.cairn.info/revue-pole-sud-2008-2-page-9.htm

Pour citer cet article :

Marcet Joan et al., « Les élections de mars 2008 en Espagne et en Catalogne »,

Pôle Sud, 2008/2 n²9, p. 9-24.

Distribution électronique Cairn.info pour ARPoS.

(c) ARPoS. Tous droits réservés pour tous pays.

La reproduction ou représentation de cet article, notamment par photocopie, n'est autorisée que dans les limites des conditions générales d'utilisation du site ou, le cas échéant, des conditions générales de la licence souscrite par votre établissement. Toute autre reproduction ou représentation, en tout ou partie, sous quelque forme et de quelque manière que ce soit, est interdite sauf accord préalable et écrit de l'éditeur, en dehors des cas prévus par la législation en vigueur en France. II est précisé que son stockage dans une base de données est également interdit. 


\title{
LES ÉLECTIONS DE MARS 2008 EN ESPAGNE ET EN CATALOGNE
}

\author{
Joan Marcet, Lucia Medina \& Marta Luque \\ Université Autonome de Barcelone \\ Institut de Ciències Polítiques i Socials
}

\section{RÉSUMÉ I ABSTRACT}

\begin{abstract}
Cet article analyse les résultats des élections législatives du 9 mars 2008, en Espagne, ainsi qu' une focale particulière sur la Catalogne. Nous examinons d'abord le contexte politique du scrutin, en prenant en compte les principaux déterminants de la législature 2004-2008 et les éléments d'innovation qui sont apparus en 2008. Nous analysons ensuite la géographie électorale, en plaçant la focale sur les phénomènes de mobilisation / démobilisation que l'on peut relever à l'échelle provinciale, ainsi que leur impact sur la physionomie de l'implantation du PP et du PSOE. Enfin, nous soulignons le rôle majeur de la Catalogne dans le résultat final.

The article analyses the electoral results of Spanish legislative elections held on 9 March 2008, stressing on the results in Catalonia. First, we examine the political context of the election, taking into account the main determinants of the 2004-2008 legislature and the innovation elements produced during the last electoral campaign. Secondly, we look at the electoral geography, focusing on the mobilization/demobilization patterns pointed out by the results at provincial level and their possible impact on the PP and PSOE figures. Finally, the article underlines the deciding role of Catalonia on the general final outcome.
\end{abstract}

\section{MOTS-CLÉ I KEYWORDS}

Campagne électorale, Catalogne, élections, Espagne, législature 2004-2008

Catalonia, elections, electoral campaign, Spain, 2004-2008 legislature 
Les élections législatives qui se sont déroulées en Espagne le 9 mars 2008 ressemblent davantage au second tour des précédentes quatre ans auparavant, le 14 mars $2004^{1}$, plutôt qu'à celles confirmant, amplifiant ou modifiant la majorité parlementaire obtenue lors du rendez-vous électoral précédent.

Leurs résultats donnent en effet clairement la victoire au Parti Socialiste, mais les différents indicateurs de ces résultats, depuis la participation jusquà la concentration du vote, n'éclairent que partiellement l'analyse électorale. Il faut donc tenir compte du contexte pré- et post-électoral de 2004 et analyser les éléments qui ont marqué la législature de 2004-2008 afin de sérier les questions clés de l'interprétation des résultats de 2008.

Parmi les dix élections législatives générales qui ont eu lieu en Espagne depuis le rétablissement de la démocratie, on peut estimer quatre d'entre elles (1979, 1986, 1989 et 2000) comme des élections « dans la continuité », caractérisées par une participation électorale plutôt faible (entre 68 et $70 \%$ ). Pour cinq autres (1977, 1982, 1993, 1996 et 2004), la participation a oscillé entre 75 et $80 \%$, coïncidant avec des alternances politiques au niveau national ou bien à des compétitions électorales vives.

Tableau 1. Résultats électoraux comparés de 2008 et de 2004

\begin{tabular}{|l|rcc|rcc|}
\hline & \multicolumn{3}{|c|}{2008} & \multicolumn{3}{c|}{2004} \\
\hline Participation & 25.900 .439 & $73,8 \%$ & & 26.155 .436 & $75,6 \%$ \\
\hline PARTI & Voix & $\%$ exprimé & Sièges & Voix & $\%$ exprimé & Sièges \\
PSOE+PSC $^{1}$ & 11.288 .689 & 43,8 & 169 & 11.026 .163 & 42,6 & 164 \\
PP+UPN $^{1}$ & 10.277 .809 & 39,9 & 154 & 9.763 .144 & 37,7 & 148 \\
IU+ICV-EUiA $^{1}$ & 969.871 & 3,7 & 2 & 1.284 .081 & 5,0 & 5 \\
CiU & 779.425 & 3,0 & 10 & 835.471 & 3,2 & 10 \\
PNB & 306.128 & 1,2 & 6 & 420.980 & 1,6 & 7 \\
ERC & 291.532 & 1,1 & 3 & 652.196 & 2,5 & 8 \\
Autres & 755.639 & 2,9 & 6 & 680.111 & 2,6 & 8 \\
\hline
\end{tabular}

${ }^{1}$ Le PSOE se présente en Catalogne sous le sigle du Partit dels Socialistes de Catalunya (PSC) ; le PP en Navarre, sous celui de I'Union del Pueblo Navarro (UPN), et I'IU, en Catalogne, sous celui d'Iniciativa per Catalunya Verds - Esquerra Unida i Alternativa (ICV-EUiA).Dans tous les cas, il s'agit de partis différents du point de vue formel.

Source: Elaboration propre à partir des données officielles de la Junte Electorale Centrale et du Ministère de I'Intérieur.

Les élections de 2008 se rapprochent davantage de ce dernier type d'élections à forte confrontation : avec $73,8 \%$ de participation, elles se situent à proximité de la participation enregistrée lors des élections de 2004 (75,7\%), distance qui se réduit même à seulement $0,4 \%$ si l'on ne prend pas en compte le vote des citoyens espagnols de l'étranger, normalement moins impliqués et moins influencés par le débat politique quotidien.

1. Pour approfondir l'analyse des résultats des élections générales du 2004, voire Montero, Lago \&Torcal (2007), Oñate \& Ocaña (2006), Colomer (2005), et Pallarés, Bosso \& Muñoz (2004). 
Cet indicateur, qui éclaire en principe le sens politique de l'élection, nous conduit à procéder ici de façon nuancée et à faire une lecture parallèle des élections de 2008 et de celles de 2004, ainsi quà analyser de manière plus précise leur environnement politique tout au long de la législature. En comparant tout simplement les données fournies pour les deux élections, deux phénomènes apparaissent clairement : la concentration du vote autour des deux principales formations au niveau national, le Partido Socialista Obrero Español (PSOE) et le Partido Popular (PP), et la progression sensible des votes pour le PP par rapport à ceux en faveur du PSOE.

En effet, comme nous l'analyserons plus loin, lors des élections de mars 2008, la polarisation s'est accrue et ceci s'est traduit dans les votes en faveur des deux principaux partis de gouvernement. Mais cette concentration, dans le cas du PP qui a gagné plus de cinq cent mille voix (soit 2,2\% de suffrages exprimés) a été supérieure à celle des socialistes, qui n'ont obtenu qu'un peu plus de deux cent soixante mille voix supplémentaires (soit 1,2\% de suffrages exprimés). C'est également le cas pour le nombre de sièges: alors que le PP en obtient six de plus qu'en 2004, les socialistes n'augmentent que de cinq sièges. Ainsi, à côté d'autres explications possibles de ces résultats, on a assisté à une réplique accentuée des résultats de 2004, que seule peut éclairer l'analyse post-électorale de ces élections et l'explicitation de ce qui a marqué le débat politique au cours des quatre années de législature.

\section{Le contexte politique: la «gueule de bois» électo- rale de 2004 et les quatre années de législature}

La mise en marche de la législature 2004-2008 a été conditionnée par une mauvaise acceptation des résultats du 14 mars 2004 de la part du PP. Ce parti, à qui tous les sondages, antérieurs à ces élections, attribuaient clairement la victoire $^{2}$, expliquera sa défaite électorale par les effets de l'attentat terroriste de Madrid, le 11 mars 2004, et par la prétendue manipulation et utilisation politique de celui-ci par le $\mathrm{PSOE}^{3}$.

La quasi totalité des études post-électorales attribuent à l'attentat de Madrid une influence sur les résultats électoraux du 14 mars et, plus précisément, sur la gestion politique et informationnelle de cet évènement par le gouvernement du PP, que ce soit parce que cela a mobilisé une part de l'électorat qui n’avait

2. La seule incertitude qui apparaissait dans la majorité des sondages concernait la portée de la victoire du PP. Seul le sondage publié par La Vanguardia une semaine avant les élections, le 7 mars 2004, élaboré par Noxa Consulting, prédisait une égalité technique entre le PP et le PSOE et la possibilité d'une défaite du PP.

3. En ce sens, le rapport entre les résultats électoraux des élections du 2004 et l'attentat terroriste, on peut consulter Lago \& Montero (2007), Torcal \& Rico (2004), Michavila (2004) et Barreiro (2004). 
pas prévu de participer au vote, ou bien parce qu'une partie des votants assez significative bien que d'une moindre magnitude - a décidé de changer le sens de son vote, spécialement du PP vers le PSOE. Létude post-électorale du Real Instituto El Cano, tout comme l'étude post-électorale du Centro de Investigaciones Sociológicas (CIS), ou encore le sondage réalisé par l'Observatorio Político Autonómico 4 (OPA), nous fournissent des données suffisamment significatives sur l'attribution de la responsabilité politique au gouvernement Aznar, que ce soit à propos des causes de l'attentat mais aussi, et plus particulièrement, de la gestion politique et informationnelle de ce dernier. Des données également précieuses quant aux changements d'attitude électorale - que ce soit en ce qui concerne la participation ou encore le sens du vote ${ }^{5}$. La controverse politique liée l'attentat terroriste de Madrid du 11 mars marquera pratiquement la totalité de la législature. Une Commission d'Enquête du Congrès des Députés continuera ses travaux pendant les deux premières années de la législature, avec un large écho médiatique, et la présence en tant que témoins, pour la première fois dans l'histoire parlementaire espagnole, du chef de gouvernement sortant, José M. Aznar et du président du gouvernement actuel, José L. Rodríguez Zapatero. Les conclusions de cette Commission, en confrontant le PP aux autres formations parlementaires, n'ont pas mis un terme à la polémique. Par la suite, le jugement de certains des auteurs matériels, des commanditaires et des complices de l'attentat a encore prolongé la confrontation, à laquelle certains médias proches du PP contribuèrent de manière plus particulière. La sentence du procès de l'attentat du 11 mars, avec de lourdes condamnations pour tous les accusés, ainsi qu'un récit et une argumentation sur les faits, interdit les spéculations sur les auteurs et les responsables de l'attentat. Cela semblait mettre un point final à une controverse politique de près de quatre ans. Il faudra cependant attendre les élections du 9 mars 2008 pour que le PP abandonne définitivement cette question comme argument de débat politique avec le PSOE.

Le débat territorial sur l'approfondissement de l'État des Autonomies constitue le deuxième élément de confrontation entre le PP et le gouvernement socialiste. Ce débat provient d'une attitude plus ouvertement favorable au caractère plurinational et pluriculturel de l'État espagnol de la part du nouveau gouvernement socialiste, dirigé par le président Rodríguez Zapatero. Il provient aussi de l'initiative, née en Catalogne, de rouvrir le débat territorial à partir de la réforme de son Statut d'Autonomie, réforme encouragée par le nouveau gouvernement de gauche, constitué après les élections de novembre 2003,

4. L’Observatorio Político Autonómico (OPA) est formé par l'Institut de Ciències Polítiques i Socials (ICPS), le Centro de Análisis y Documentación Política y Electoral de Andalucía (CADPEA), l'Université de Saint-Jacques de Compostelle et l'Université du Pays Basque. 5. Voir le baromètre de juin 2004 du Real Instituto El Cano, l'étude post-électorale du CIS (mars-avril 2004) et «l'Enquête post-électorale du 14-M-2004», réalisée parl'Observatorio Político Autonómico (www.opa151.com). 
et appuyée par la quasi totalité des forces politiques catalanes à l'exception du PP. Le débat en Catalogne et dans le reste de l'Espagne sur le nouveau Statut d'Autonomie catalan sera utilisé par le PP pour alimenter un débat politique sans précédent, bien que le même $\mathrm{PP}$ apporte parallèlement son appui à des réformes statutaires dans d'autres territoires (Îles Baléares, Andalousie ou Aragon).

Ce débat territorial s'ajoutera au milieu de la législature à l'utilisation politique partisane - ce qui est également sans précédent - du débat sur la lutte contre le terrorisme d'ETA. La déclaration unilatérale d'une trêve de la part de la bande terroriste en mars 2006 permettra toutes sortes d'accusations de la part du PP, dénonçant la connivence politique du gouvernement PSOE avec l'ETA. À ces accusations s'adjoindront celles des associations de victimes du terrorisme qui provoqueront, pendant et après la trêve, de nombreuses manifestations pour protester contre de possibles conversations politiques entre le gouvernement et la bande terroriste. Mais au-delà des opportunités et des débats autour de la trêve d'ETA, la politique anti-terroriste développée par le gouvernement a constitué, pour la première fois dansl'histoire démocratique espagnole, un élément de controverse et d'utilisation partisane de la part du PP. Cette attitude des populaires, relayée par les médias écrits et audiovisuels les plus proches de ce parti, constitue l'une des clés de la géographie des résultats des élections de mars 2008. Les territoires les plus affectés par le débat autonomique et par le débat sur la politique antiterroriste-comme nous le verrons plus loin, la Catalogne et Euskadi -s'opposent, en termes de soutien électoral au PP, à d'autres territoires où le discours populaire a trouvé une meilleure audience. Les positions les plus nationalistes espagnoles arborées par le PP s'opposeront dans le débat politique et électoral à une attitude plus ambiguë de la part du PSOE, qui va d'un nationalisme « espagnoliste » modéré jusqu'au fédéralisme.

Le troisième élément de confrontation entre les deux principaux partis au cours de la législature s'est produit à partir des réformes mises en œuvre par le gouvernement socialiste sur le terrain des droits civils. Laccélération des formalités pour le divorce, la légalisation du mariage entre homosexuels ou la législation tendant à favoriser l'égalité entre hommes et femmes ont aussi constitué des éléments de confrontation politique entre des visions politiques opposées entre le PP et le PSOE. L'intervention de la hiérarchie de l'Église catholique a également contribué à rendre cette confrontation plus visible. Pour la première fois dans l'histoire récente de l'Espagne, des évêques et des cardinaux de l'Église catholique participaient à des manifestations citoyennes organisées par diverses entités et avec l’appui du PP. Ce positionnement de la hiérarchie ecclésiastique culminait peu de semaines avant les élections avec un très grand rassemblement à Madrid en faveur de «la famille traditionnelle», lancé par l'épiscopat lui-même. 
La législature a aussi été marquée par une situation économique au beau fixe, qui commençait seulement à se dissiper dans les semaines immédiatement antérieures aux élections, et dont le gouvernement a profité pour réaliser certaines avancées importantes en matière de politiques sociales, tout en profitant de la marge de manœuvre que cette situation lui permettait. La tentative du PP et de son candidat à la présidence du gouvernement, Mariano Rajoy, de profiter des premiers indices de crise de l'économie, au niveau global comme au niveau espagnol, s'est heurtée à la résistance, de la part des socialistes, à entrer dans le débat sur un quelconque signe de ralentissement ou de crise économique. Les indicateurs des premiers mois de 2008 donnaient encore l'avantage aux responsables économiques gouvernementaux face aux argumentations plus pessimistes exprimées par les dirigeants du PP. Tout au long de ces quatre années de législature, diverses consultations électorales ont également eu lieu, certaines au niveau de l'État et d'autres au niveau des Autonomies, qui ont eu une incidence sur le débat politique de la période que nous analysons. En juin 2004 avaient lieu les élections au Parlement Européen, dont le résultat, malgré la faible participation enregistrée $(45,1 \%)$ semblaient corroborer les résultats des élections législatives du mois de mars : le PSOE gagnait ces élections avec 43,4\% malgré le regain du PP (41,2\%) par rapport aux élections générales de mars. Malgré ces résultats, comme nous l'avons déjà souligné, le PP s'est maintenu dans la posture du refus d'accepter politiquement sa défaite aux élections législatives, trois mois auparavant.

Pendant l'année 2005, des élections autonomiques ont eu lieu en Euskadi et en Galice. Dans le premier cas, c'est le retour à une carte électorale traditionnelle : le Partido Socialista de Euskadi-PSOE récupère la deuxième place, à une distance notable de la coalition nationaliste gagnante formée par le Partido Nacionalista Vasco-Eusko Alkartasuna (PNV-EA), le PP étant à nouveau relégué en troisième position. En Galice, la perte de la majorité absolue de la part du PP a permis la formation, pour la première fois, d'un gouvernement de coalition entre le Partido de los Socialistas de Galicia-PSOE et les nationalistes du Bloque Nacionalista Galego (BNG). Ces deux élections, dans le cadre territorial autonomique, reflétaient donc un certain recul du PP et une consolidation des socialistes.

En novembre 2006 de nouvelles élections ont lieu en Catalogne. Malgré le recul général de l'ensemble des partis politiques catalans, avec un très faible taux de participation électorale (56\%), les résultats permettent le maintien du gouvernement de gauche conduit par le Parti Socialiste (PSC) et situent à nouveau le $\mathrm{PP}$ en quatrième rang, dépassé de très peu par les anciens communistes et éco-socialistes d'ICV-EUiA.

En mai 2007, se déroulent les élections municipales en Espagne et des élections autonomiques dans treize Communautés Autonomes. Le résultat de ces élections, que certains analystes politiques voulaient considérer comme un 
signe des élections générales suivantes, montrait l'équilibre existant entre les deux principales forces politiques. Aux élections municipales, le PP l'emportait de justesse dans l'ensemble de l'État, avec $0,7 \%$ des voix en plus, alors que le PSOE obtenait le plus grand nombre de conseillers municipaux. Mais aucune des principales villes espagnoles ne changeait de majorité municipale ${ }^{6}$. Aux élections autonomiques, les deux forces politiques maintenaient globalement leur implantation territoriale : le PP se renforce et accroît son avance sur le PSOE à Madrid et dans la Communauté Valencienne mais il perd le gouvernement des Îles Baléares. N'y disposant plus de la majorité absolue, il permet la formation d'un gouvernement de coalition de partis de gauche et nationalistes, dirigé par le PSOE. Aux Îles Canaries, bien qu'il perde les élections, le PP réussit à maintenir sa participation au gouvernement autonomique en coalition avec les nationalistes canaris. Ces résultats territoriaux des élections autonomiques apparaissent comme un signe de la répartition territoriale des résultats des élections législatives générales de mars 2008.

\section{Une campagne électorale longue et avec de nouveaux formats}

Malgré l'intensité et la crispation caractéristiques de la législature que nous venons de rappeler, les différents baromètres et sondages d'opinion réalisés pendant ces quatre années situaient toujours le PSOE en avance sur le PP en termes d'intentions de vote ${ }^{7}$. Cette situation semblait annoncer une campagne longue, intense et avec des éléments nouveaux, ce qui s'est finalement passé. Bien que la date des élections coïncide avec le terme légal de la législature, au mois de janvier 2008, on peut considérer que la ratification « in extremis 》 des budgets généraux de l'État pour 2008 lors de l'automne précédent, la sentence du procès sur les attentats du 11 mars, également prononcée au cours de l'automne, ainsi que les premiers indices de ralentissement économique signalaient le début d'une campagne longue et, dans une certaine mesure, une reproduction des stratégies conduites par les différents acteurs politiques, plus spécialement le PP et le PSOE, tout au long de la législature.

$\mathrm{Au}$ fur et à mesure que l'échéance proprement dite se rapprochait, deux nouveautés sont apparues par rapport aux campagnes précédentes. D’un côté, un accord entre toutes les forces politiques parlementaires a vu le jour, sans crier gare, pour modifier le format de présentation des différents messages politiques

6. La seule exception se situe en Catalogne, où, dans le duel qui les opposent aux nationalistes catalans modérés de $\mathrm{CiU}$, les socialistes ont réussi à gagner la mairie d'une capitale provinciale, Tarragone.

7. Voir l'évolution de l'intention de vote pour le PP et le PSOE pour les élections générales dans les baromètres du CIS à l'adresse: http://www.cis.es/cis/opencms/ES/2_barometros/ Indicadores_PI/documentos/B606050010a.html. 
dans les médias audiovisuels publics. La législation électorale comportait l'interdiction de la publicité électorale dans les radios et les télévisions publiques, que ce soit au niveau de l'État ou des autonomies, et la fourniture d'espaces gratuits de campagne, diffusés avec une bande-annonce au début et à la fin, juste après les journaux télévisés. L'accord auquel les partis sont parvenus, accepté par la Junte électorale centrale, a converti ces espaces gratuits en de véritables spots publicitaires de courte durée, diffusés à n'importe quel moment de la programmation de radios et de télévisions publiques ${ }^{8}$. La deuxième nouveauté de la campagne, beaucoup plus médiatisée en ce qui concerne ses négociations et ses réalisations cette fois, a consisté en la réalisation de deux débats en « face à face » à la télévision (sur les chaines privées en l'occurrence) entre les deux principaux candidats à la présidence du gouvernement, le socialiste Rodríguez Zapatero et le populaire Mariano Rajoy. Tout au long de plus de trente années d'élections législatives générales en Espagne, seuls deux débats électoraux de ce type avaient eu lieu, lors de la campagne de 1993, qui avaient mis face à face Felipe González et José M. Aznar. Quinze années avaient été nécessaires, ainsi qu'une législature caractérisée par une forte compétition et une proximité entre les deux premières offres politiques pour que ces débats électoraux se produisent à nouveau.

Les deux débats, assez lents du point de vue technique et aux contenus politiques excessivement réglés et prévisibles, par rapport à d'autres débats à deux ou plusieurs candidats, offraient une réelle image de bipolarisation électorale ainsi qu'une image irréelle de suffisance de la candidature socialiste. En fait, les différents sondages électoraux, publiés entre début janvier 2008 et la semaine précédant les élections, présentaient toujours le PSOE comme vainqueur des élections avec un avantage oscillant entre trois et six pour cent ${ }^{9}$. Or le véritable résultat des élections du 9 mars a situé les socialistes à seulement quatre points du PP.

\section{La carte électorale résultant des élections : consolidation territoriale et bipartisme en augmentation}

Une lecture générale des résultats dépasse ce qui avait été annoncé au début de cet article concernant la victoire socialiste, claire mais étroite, et nous permet

8. Si législation électorale espagnole interdit la publicité électorale sur les chaînes de télévision privées, ce n'est pas le cas des stations de radio privées.

9. Pour la première fois également, bénéficiant d'un vide juridique, le journal El Periòdico de la Catalogne, a profité de son édition dans la Principauté d'Andorre, et par ailleurs du site web de cette édition, pour échapper à l'interdiction de publication de sondages pendant les cinq jours précédant la date des élections et en a même publié jusqu’à la veille au soir de ces élections. 
de situer deux des principaux éléments d'analyse de ces élections de mars 2008 : la consolidation des implantations territoriales des deux principales forces politiques, et l'augmentation du bipartisme jusquà des proportions inconnues jusqualors.

La consolidation des implantations territoriales, analysée et élargie plus loin, avait pour précédent les élections de 2004 ainsi que, en partie, les élections autonomiques de 2007. Le PSOE progresse de manière sensible dans les deux communautés insulaires, les Canaries et les Baléares, ainsi qu’aux Asturies. Il s'impose, avec un résultat remarquable, au Pays Basque, en Aragon et surtout en Catalogne, et il recule en Castille-La Mancha et plus encore en Andalousie. De son côté, le PP consolide sa position dans une frange centrale qui va de la Galice à l'Est de l'Espagne, en accroissant plus particulièrement son influence à Madrid et dans la Communauté Valencienne. Cette carte électorale, que nous analyserons plus loin au niveau des circonscriptions provinciales, montre les faiblesses de chacun des deux partis plutôt que leurs forces respectives. Le PP semble avoir réussi à faire une percée électorale grâce à sa stratégie nationaliste espagnole, assortie d'une position solide dans certaines communautés qu'il gouverne. Il ne réussit pas, par contre, à progresser auprès de l'électorat du centre du pays, tout spécialement dans les communautés les plus dynamiques d'un point de vue économique et politique. Le PSOE quant à lui montre de grandes difficultés à maintenir l'équilibre entre un discours autonomiste pseudo fédéral et les pulsions internes et externes de caractère plus nationaliste espagnol. Il subit en même temps les conséquences de sa déstructuration organisationnelle dans certaines communautés, comme Madrid et Valence. En Andalousie, le recul du PSOE et la progression du PP peuvent s' interpréter à la fois en termes d'usure électorale, après trente années de victoires continuelles aux élections autonomiques et nationales et, plus spécifiquement, en termes d'équilibre et de confrontation avec le PP, si l'on analyse plus en détail la situation géographique du recul (plus concrètement Almeria et Malaga).

Le deuxième élément d'analyse globale évoqué est en relation avec l'accentuation d'un phénomène qui n'est pas nouveau dans le panorama électoral espagnol : le bipartisme. Si l'on tient compte de toute la séquence d'élections générales, de 1977 à 2004, on voit que la somme des deux premiers partis, en pourcentages des suffrages exprimés, a progressivement augmenté de 63,7\% en 1977 à $80,3 \%$ en 2004. Si l'on considère les sièges obtenus par les deux premiers partis comme une déformation produite par le système électoral, le pourcentage a toujours été situé au-dessus de la barre des $80 \%$, arrivant même en 2004 à $89,1 \%$. La simple observation du tableau des résultats électoraux, confrontant ceux de 2004 à ceux de mars 2008 , nous fait voir l'accentuation de cette tendance. Le total des pourcentages de suffrages exprimés obtenus par le PSOE et le PP atteint 83,75\% et le pourcentage de sièges (322 sur 350 ) 92\%. Tous ces résultats se produisent évidemment au détriment des autres partis 
minoritaires, qui ont tous perdu en pourcentage de suffrages et en sièges ${ }^{10}$, et ce de manière encore plus notable pour Izquierda Unida-Iniciativa per Catalunya Verds (IU-ICV) et Esquerra Republicana de Catalunya (ERC). Il semble clair que la forte polarisation en faveur des PSOE-PP pendant toute la législature, ainsi qu'une campagne très bipolarisée ont favorisé les deux principaux partis qui ont bénéficié d'une remarquable concentration des suffrages exprimés en leur faveur, provenant davantage du reste de formations politiques que d'un déplacement de suffrages entre ces deux partis, lequel semble avoir été favorable au PP, selon les données fournies par les sondages post-électoraux ${ }^{11}$.

\section{La répartition territoriale du vote : signes de mobilisation et de démobilisation}

Comme nous l'avons déjà signalé, les résultats électoraux des élections générales de 2008 marquent une certaine continuité par rapport à ceux des élections de 2004, ce qui a favorisé aussi la consolidation du vote d'un point de vue territorial. Lorsqu'on examine quel parti a reçu le plus de suffrages dans chaque circonscription provinciale, la carte de la répartition du vote de 2008 est très similaire à celle de 2004. Les deux forces politiques majoritaires, le PSOE et le PP continuent, pratiquement, à s'imposer toujours dans les mêmes provinces et ce dans leur totalité. Ce sont des provinces qu'ils avaient déjà remportées en 2004, et le paysage électoral est fait d'espaces électoraux contrastés. Le PSOE continue, dans les grandes lignes, à être le parti qui reçoit le plus de votes en Andalousie, en Aragon, en Catalogne et en Extremadura, alors que le $\mathrm{PP}$ est le parti qui remporte le reste, dans une frange géographique qui commence en Galice et qui s'étend jusqu'au levant de l'Espagne. Néanmoins, derrière cette continuité, de petits changements émergent qui pourraient bien être significatifs. En effet, même si le PP continue à être le parti qui reçoit le plus de suffrages dans le plus grand nombre de circonscriptions électorales (28 sur 52), au cours de ces élections, il a perdu, en faveur du PSOE, cette position hégémonique dans trois provinces (Asturies, Îles Baléares et Las Palmas). Cette perte est cependant partiellement compensée par la victoire du PP à Almeria et à Ciudad Real, auparavant aux mains des socialistes.

D'autre part, le PSOE a réussi à réduire la différence qui le séparait du PP en ce qui concerne le nombre de provinces gagnées : en 2004, il a été la force recevant le plus de suffrages dans 21 des 52 circonscriptions, et pour les élections de 2008, celui obtenant une majorité de suffrages dans 24 provinces sur 52 . Il faut par ailleurs remarquer la victoire du PSOE à Guipuscoa et à Biscaia, qui étaient auparavant aux mains de la coalition nationaliste formée par PNV-EA.

$\overline{10 .}$ Seul CiU a maintenu ses 10 sièges bien que cette formation ait perdu $0,2 \%$ au niveau de l'État.

11. Voir par exemple l'étude post-électorale no $2.757 \mathrm{du}$ CIS. 
Carte 1. Élections générales 2004 et 2008 :

Parti ayant reçu le plus de votes dans chaque circonscription provinciale (pourcentage de suffrages exprimés).
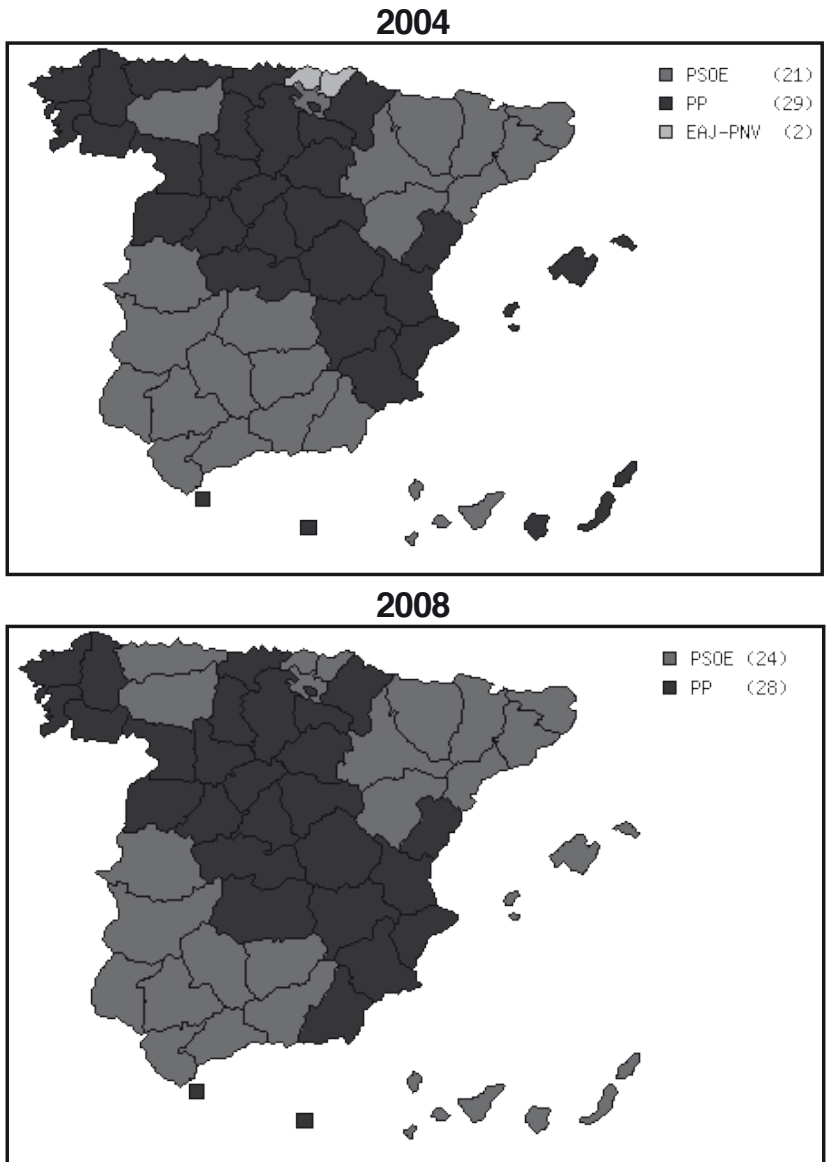

Source: http://electionresources.org

Cependant, cette consolidation de la répartition territoriale du vote ne doit pas nous faire perdre de vue que, derrière une apparente stabilité, se dissimulent certains changements quant au degré de mobilisation des électeurs respectifs des deux partis majoritaires. Ce constat met en évidence leurs forces mais surtout leurs faiblesses électorales et, à moyen terme, ces mutations peuvent induire une transformation partielle ou même substantielle de la carte électorale. Dans ce sens, sur les tableaux 2 et 3, les provinces sont classifiées en fonction des résultats électoraux obtenus en 2008 par chaque parti par rapport aux résultats de 2004, ceci mis en perspective avec les diminutions et les augmentations correspondant à la participation. Dans le premier quadrant, 
en nuance de couleur gris clair figurent les circonscriptions dans lesquelles chaque parti mobilise plus de suffrages, et dans le dernier quadrant, en nuance de couleur gris foncé, les provinces où chaque parti subit une démobilisation. L'examen des tableaux permet donc d'arriver à certaines conclusions.

Tout d'abord, on peut considérer que, bien que le PSOE soit la force recueillant le plus grand nombre de suffrages et la plus importante proportion de pourcentages de suffrages exprimés au niveau de l'État, le PP bénéficie le plus de l'augmentation de l'affluence aux urnes, là où elle s'est produite. En effet, leur pourcentage de vote par rapport au corps électoral augmente dans 11 circonscriptions provinciales, alors que celui des socialistes ne le fait que dans 5 circonscriptions. C'est dire que là où la participation augmente, le PSOE mobilise moins que le PP. En outre, cette progression en faveur des socialistes ne suit aucun schéma territorial défini, alors que celle en faveur des populaires, touchant principalement Madrid, Murcie et les provinces de la Communauté Valencienne, permet d'envisager le déploiement d'un certain effet de «contagion» du vote autonomique sur les résultats des élections générales dans ces provinces. Ceci étant donné que l'ensemble d'entre elles appartiennent à des communautés autonomes gouvernées par le PP - bilan qui devrait en tout cas être vérifié de manière adéquate. D'autre part, les populaires augmentent aussi leur pourcentage de suffrages dans quatre provinces de Castilla-La Mancha, alors que le PSOE fléchit, ce qui met en évidence les difficultés des socialistes à tirer parti de la légère augmentation de participation dans cette communauté.

Ensuite, bien que le PSOE fasse preuve d'une moindre capacité mobilisatrice, appréciée à partir du nombre de provinces où il gagne des suffrages, il est certes vrai que c'est aussi le parti qui a subi la démobilisation la plus faible, évaluée également de la même manière. A savoir, dans les circonscriptions où la participation a baissé, le PSOE n'a subi une érosion de suffrages que dans 11 provinces, alors que le PP a faibli dans 24 provinces. La démobilisation du vote socialiste affecte principalementl'Andalousie, alors que pour le PP, elle se produit dans les communautés historiques ayant des partis nationalistes plus ou moins forts (Galice, Catalogne, Pays Basque et Navarre), mais aussi en Aragon, en Asturies, en Extremadura, aux Îles Baléares et dans certaines parties de Castille et Léon. Tous ces signes de démobilisation, que ce soit pour le PSOE ou pour le PP, sont précisément ceux qui laissent présager de futures transformations possibles de la carte électorale, dans la mesure où l'on entrevoit les difficultés des socialistes et des populaires à maintenir leurs niveaux de votes dans certaines zones déterminées (dans le cas du PSOE, en Andalousie, et dans le cas du PP, surtout en Castille et Léon). 
Tableau 2. Marques de mobilisation et de démobilisation électorale du PP aux élections générales de 2008, par rapport à 2004 .

\begin{tabular}{|c|c|c|}
\hline & $\begin{array}{c}\text { Augmentation du vote pour } \\
\text { le PP }\end{array}$ & $\begin{array}{c}\text { Diminution du vote pour } \\
\text { le PP }\end{array}$ \\
\hline $\begin{array}{l}\text { La participation augmente } \\
\text { par rapport aux élections } \\
\text { générales de } 2004\end{array}$ & $\begin{array}{l}\text { Albacete Alicante Almería } \\
\text { Ciudad Real Cuenca Madrid } \\
\text { Melilla Murcie Ségovie } \\
\text { Tolède Valence (11) }\end{array}$ & $\begin{array}{l}\text { Ávila Orense Teruel Zamora } \\
\text { (4) }\end{array}$ \\
\hline $\begin{array}{l}\text { La participation diminue par } \\
\text { rapport aux élections généra- } \\
\text { les de } 2004\end{array}$ & $\begin{array}{l}\text { Cadiz Castellón Cordoue } \\
\text { Granada Guadalajara Huelva } \\
\text { Jaén Málaga Navarre Séville } \\
\text { Sta. Cruz de Tenerife Valla- } \\
\text { dolid Saragosse (13) }\end{array}$ & $\begin{array}{l}\text { A Coruña Alava Asturies } \\
\text { Badajoz Barcelone Burgos } \\
\text { Caceres Cantabrie Ceuta } \\
\text { Gérone Guipúzcoa Huesca } \\
\text { îles Baléares Las Palmas } \\
\text { La Rioja León Lérida Lugo } \\
\text { Palencia Pontevedra Sa- } \\
\text { lamanque Soria Tarragone } \\
\text { Bizcaya (24) }\end{array}$ \\
\hline
\end{tabular}

Source: élaboration propre à partir des données du ministère de l'Intérieur.

Tableau 3. Marques de mobilisation et de démobilisation électorale du PSOE aux élections générales de 2008, par rapport à 2004.

\begin{tabular}{|l|l|l|}
\hline & $\begin{array}{l}\text { Augmentation du vote pour } \\
\text { le PSOE }\end{array}$ & $\begin{array}{c}\text { Diminution du vote pour le } \\
\text { PSOE }\end{array}$ \\
\hline $\begin{array}{l}\text { La participation augmente } \\
\text { par rapport aux élections } \\
\text { générales de 2004 }\end{array}$ & $\begin{array}{l}\text { Ávila Melilla Orense Teruel } \\
\text { Zamora (5) }\end{array}$ & $\begin{array}{l}\text { Albacete Alicante Almería } \\
\text { Ciudad Real Cuenca Madrid } \\
\text { Murcie Ségovie Tolède } \\
\text { Valence (10) }\end{array}$ \\
\hline $\begin{array}{l}\text { La participation diminue par } \\
\text { rapport aux élections généra- } \\
\text { les de 2004 }\end{array}$ & $\begin{array}{l}\text { A Coruña Alava Asturies } \\
\text { Badajoz Barcelone Burgos } \\
\text { Caceres Cantabrie Ceuta } \\
\text { Gérone Guipúzcoa Huesca } \\
\text { Îles Baléares Las Palmas } \\
\text { León Lérida Lugo Navarre } \\
\text { Palencia Pontevedra Sala- } \\
\text { manque Soria Sta. Cruz de } \\
\text { Tenerife Tarragone Bizcaya } \\
\text { Saragosse (26) }\end{array}$ & $\begin{array}{l}\text { Cadiz Castellón Cordoue } \\
\text { Granada Guadalajara Huelva } \\
\text { Vaén La Rioja Málaga Séville }\end{array}$ \\
Vallid (11)
\end{tabular}

Source: élaboration propre à partir des données du ministère de l'Intérieur.

\section{Les élections en Catalogne : des résultat décisifs}

Historiquement, la Catalogne a toujours été un espace essentiel au Parti Socialiste au niveau national. Depuis 1977, le Partit dels Socialistes de Catalunya (PSC), parti indépendant du PSOE mais attaché du point de vue programmatique et organique à ce dernier, a été le parti recueillant le plus de suffrages à toutes les élections au Congrès des Députés. Cependant, certains moments de tension entre les gouvernements de l'État et les gouvernements autonomiques (les deux étant gouvernés par des socialistes) lors des quatre 
dernières années ont généré des doutes sur ce que seraient les résultats électoraux dans cette communauté, et sur leur impact en Espagne plus généralement. Pour cette raison, les élections législatives de 2008 en Catalogne ont eu lieu dans un climat d'incertitude concernant l'évolution du vote socialiste.

La législature 2004-2008 a été marquée par les situations de tension entre le gouvernement de l'État et le gouvernement de la Generalitat, tensions qui ont eu des répercussions sur les relations entre le PSC et le PSOE. Ces confrontations ont principalement concerné le débat sur le modèle territorial de l'État et le processus d'élaboration, de négociation et de ratification de la réforme du nouveau Statut d'Autonomie. Plus récemment, des problèmes ont également surgi à propos de la négociation du modèle de financement de ce dernier, ou de la crise des infrastructures, qui ont ravivé le débat sur les investissements de l'État en Catalogne. Ces éléments de tension et de confrontation affectaient principalement les perspectives électorales du PSC. Pour cette raison, la campagne électorale socialiste s'est évertuée à souligner les dangers d'une possible victoire du PP, au lieu de se centrer sur les actions menées à terme par le gouvernement du PSOE lors des quatre dernières années. Les élections législatives de 2008 en Catalogne ont pourtant été des élections de continuité, avec une répartition territoriale du vote très similaire aux élections de 2004 ou au comportement du reste de l'État, bien qu'avec certaines nuances (voir le tableau 4).

Tableau 4: Résultats électoraux comparés de 2008 et de 2004

\begin{tabular}{|l|c|c|c|c|c|c|}
\hline & & $\mathbf{2 0 0 8}$ & & & $\mathbf{2 0 0 4}$ & \\
\hline Participation & 3.743 .360 & $70,3 \%$ & & 4.032 .589 & $75,9 \%$ & \\
\hline PARTI & Voix & $\% /$ exprimé & Sièges & Voix & $\% /$ exprimé & Sièges \\
\hline PSC & 1.689 .911 & 45,4 & 25 & 1.586 .748 & 39,5 & 21 \\
\hline CiU & 779.425 & 20,9 & 10 & 835.471 & 20,8 & 10 \\
\hline PP & 610.473 & 16,4 & 8 & 638.902 & 15,6 & 6 \\
\hline ERC & 291.532 & 7,8 & 3 & 652.196 & 15,9 & 8 \\
\hline ICV-EUiA & 183.338 & 4,2 & 1 & 234.790 & 5,8 & 2 \\
\hline
\end{tabular}

Source: élaboration propre à partir des données officielles de l'Assemblée Électorale Centrale et du Ministère de l'Intérieur.

On peut tirer diverses conclusions de ces élections. En premier lieu, en ce qui concerne la participation, cette dernière a diminué de près de $6 \%$ et elle se situe parmi les plus faibles depuis 1977. Cette caractéristique est commune aux quatre circonscriptions électorales, Barcelone et Tarragone étant les deux circonscriptions présentant une baisse de participation légèrement inférieure à la diminution moyenne en Catalogne. Il faut cependant souligner qu'en Catalogne, la participation électorale aux élections pour le Congrès des Députés se situe toujours au-dessous de la participation moyenne dans l'ensemble de l'État. 
En deuxième lieu, ces élections ont accentué la tendance à la polarisation du vote autour des deux principaux partis catalans, bien que différemment par rapport à ce qui se produit dans le reste du pays. Il faut tenir compte du fait que le système de partis catalan a toujours été caractérisé par le multipartisme, dans la mesure où cinq partis y sont en concurrence. Bien qu'il soit vrai que ces élections aient consolidé une certaine tendance au bipartisme, celui-ci se produit entre le PSC et Convergència i Unió ( $\mathrm{CiU}$ ), et non avec le le $\mathrm{PP}$, très minoritaire en Catalogne : en 2004, il est passé de la troisième à la quatrième place en pourcentage de suffrages exprimés. PSC et CiU concentrent 66,5\% des suffrages exprimés, six points de plus que lors des élections de 2004. Par rapport au reste de l'État, la nuance est que dans ce cas, la différence entre les deux formations est plus grande que celle existant entre le PSOE et le PP. Comme on peut l'observer dans le tableau 4, CiU n'a pas vu son pourcentage de suffrages accru lors de ces élections, mais c'est au contraire le PSC qui a substantiellement amélioré son résultat par rapport à celui de 2004 et qui a obtenu ces six points de plus qui marquent la différence ; c'est-à-dire qu'il s'agit d'une certaine tendance au bipartisme qui n'est pas aussi équilibré que dans le reste de l'État, mais orienté en faveur des socialistes.

En corrélation avec cet élément se produit le recul électoral d'Esquerra Republicana de Catalunya (ERC) et d'Iniciativa per Catalunya Verds-Esquerra Unida i Alternativa (ICV-EUiA), et plus particulièrement pour les premiers. Alors que ICV-EUiA n'a perdu qu'un point de pourcentage de suffrages exprimés, ERC a subi un recul beaucoup plus marqué ( $8 \%$ de moins), quil'a fait passer de 8 à 3 députés, c'est-à-dire que les deux autres associés du PSC dans le gouvernement autonomique ont subi une perte de suffrages considérable lors de ces élections par rapport à celles de 2004. De toute manière, il faut introduire quelques nuances entre les deux formations. Bien que la perte de votes d'ERC soit beaucoup plus importante que celle d'ICV, la formation républicaine continue d'obtenir ses deuxièmes meilleurs résultats électoraux depuis 1977. En revanche, les éco-socialistes subissent un moindre recul, mais ils obtiennent des résultats similaires aux pires résultats obtenus au courant de leur histoire électorale, ceux de 1982.

Tout ceci nous amène à une autre conclusion : l'extraordinaire victoire du PSC lors de cette compétition électorale. Au vu des résultats, et après avoir dépassé $45 \%$ des suffrages exprimés (presque les mêmes résultats que ceux de 1982, qui furent les meilleurs de toute la série), les élections de 2008 ont balayé les doutes concernant l'amélioration ou non de ses résultats par le PSC après les conflits entre gouvernement catalan et espagnol tout au long de la législature.

En définitive, les élections de 2008 en Catalogne ont été des élections de continuité qui ont renforcé la tendance au bipartisme orienté dans ce cas en faveur du PSC, qui a de nouveau reléguéle PP loin derrière sur la scène politique catalane, et qui ont entraîné un recul considérable des partis minoritaires. 
De même, ces résultats ont également été décisifs pour l'ensemble de l'État parce qu'ils ont assuré presque mathématiquement au PSOE la majorité électorale.

\section{Références / References}

Barreiro B., «14-M: elecciones a la sombra del terrorismo», Claves de Razón Práctica, $n^{\circ}$ 141, 2004.

Colomer J. M., « The General Election in Spain, March 2004 », Electoral Studies, $n^{\circ} 24,2005$.

Torcal M. \& Rico G., « The Spanish General Election: In the Shadow of Al-Qaeda? », South European Society and Politics, vol. 9, n 3, 2004.

Michavila N., «Guerra, terrorismo y elecciones: incidencia electoral de los atentados islamistas en Madrid», Madrid, Real Instituto Elcano, Working Paper 13, 2004.

Montero J. R., Lago I. \& Torcal M. (eds.), Elecciones generales 2004, Madrid, Centro de Investigaciones Sociológicas, 2007.

Montero J. R., Lago I., « 11-M al 14-M: Terrorismo, gestión del gobierno y rendición de cuentas », in Montero J. R., Lago I. \& Torcal M. (eds.), Elecciones generales 2004, Madrid, Centro de Investigaciones Sociológicas, 2007.

Ocaña F. A. \& Oñate P., «Las arenas electorales en España y la normalidad de la convocatoria de marzo de 2004», in Molins J. \& Oñate P. (eds.), Elecciones y comportamiento electoral en la España multinivel, Madrid, Centro de Investigaciones Sociológicas, 2006.

Pallarès F., Bosso A. \& Muñoz J., « Las elecciones generales de 2004 en España », in Instituto de Derecho Público, Informe Comunidades Autónomas 2004, Barcelona, Instituto de Derecho Público, 2004. 